\title{
Artificially intelligent nanopore for rapid SARS-CoV-2 testing
}

By manufactur-
ing nanopores
with a diameter
suitable for
SARS-CoV-2
sensing and
by using a
machine
learning
algorithm,
we could make
a diagnostic
device that
allows selective
and rapid
SARS-CoV-2
detection with
high accuracy

High-accuracy detection of SARS-CoV-2 is at the heart of our efforts in tackling the ongoing coronavirus pandemic. Reverse transcription-polymerase chain reaction (RT-PCR) is currently the gold standard for viral RNA detection in saliva samples. However, RT-PCR requires timeconsuming RNA extraction and purification steps, limiting the possibility of high-throughput testing. Now, reporting in Nature Communications, Masateru Taniguchi, Yoshiharu Matsuura, Kazunori Tomono and colleagues developed an artificially intelligent nanopore device that allows SARS-CoV-2 detection with high sensitivity and specificity within 5 minutes.

Nanopore-based sensors enable the detection and analysis of viruses, without requiring viral genome extraction. Here, an electric field is applied to transport a sample through the nanopore by electrophoresis, which causes

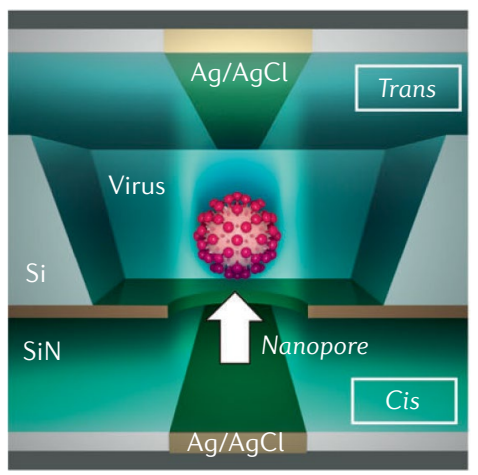

Credit: Reprinted from Taniguchi, M. et al. Combining machine learning and nanopore construction creates an artificial intelligence nanopore for coronavirus detection. Nat. Commun. 12, 3726 (2021), CC BY 4.0 (https://creativecommons.org/licenses/by/4.0/) temporal fluctuations in the ionic current that depend on the features of the sample. Thus, by analysing the ionic current versus time waveform, one can obtain information about the analytes' volume, structure and surface charge - properties that differ between different viruses.

To achieve a selective readout, that is, to automatically detect a specific virus in a given sample, the waveform data can further be classified using artificial intelligence. However, to apply machine learning algorithms for waveform classification, nanopores need to be fabricated with very high accuracy and reproducibility.

"Artificial intelligence explores the biggest differences in ionic current waveforms, and therefore, large differences in nanopore diameters can cause false readouts, that is, the algorithm identifies structural differences in the nanopores rather than in viruses," explains Tomono.

To enable accurate readouts, the researchers engineered a silicon chip featuring plastic channels and nanopores with diameters of $300 \mathrm{~nm}$ (for comparison, the diameters of coronaviruses are in the range of $\sim 80-120 \mathrm{~nm})$. Importantly, the nanopores could be precisely and reproducibly fabricated, with a diameter error of $\pm 10 \mathrm{~nm}$, as well as high yield (90\%). $\mathrm{Ag} / \mathrm{AgCl}$ electrodes were further added to each channel to allow stable current measurement with high reproducibility.

The performance of the nanopore device was first evaluated using polystyrene nanoparticles and cultivated coronaviruses, including $\mathrm{HCoV}-229 \mathrm{E}, \mathrm{MERS}-\mathrm{CoV}$, SARS-CoV and SARS-CoV-2. The resulting current-time waveforms were then used to train a random forest algorithm and to create a classification model that can distinguish between the different coronaviruses.

To generate training features for clinical samples, the ionic current-time traces of SARS-CoV-2 PCR-positive and PCR-negative saliva specimens were analysed. Using the same random forest algorithm classifier obtained in the training process, PCR-positive waveforms could be distinguished from PCR-negative waveforms in clinical samples, with a sensitivity and specificity of $90 \%$ and $96 \%$, respectively. Importantly, the assay can be performed within 5 minutes, outperforming RT-PCR tests, which usually take hours.

"By manufacturing nanopores with a diameter suitable for SARS-CoV-2 sensing and by using a machine learning algorithm, we could make a diagnostic device that allows selective and rapid SARS-CoV-2 detection with high accuracy," says Tomono.

The next step is to test the applicability of the device for other viruses and bacteria. The researchers will also apply to obtain regulatory approval for this new coronavirus test.

Christine Horejs

ORIGINAL ARTICLE Taniguchi, M. et al. Combining machine learning and nanopore construction creates an artificial intelligence nanopore for coronavirus detection. Nat. Commun. 12, $3726(2021)$ 